\title{
Performance Simulation of Non-reliable Servers in Finite-Source Cognitive Radio Networks with Collision
}

\author{
Hamza Nemouchi and Janos $\operatorname{Sztrik}^{(凶)}$ \\ Faculty of Informatics, University of Debrecen, Debrecen, Hungary \\ nemouchih@gmail.com, sztrik.janos@inf.unideb.hu
}

\begin{abstract}
This paper introduces a finite-source retrial queueing system which models cognitive radio networks. We assume two non-independent frequency bands servicing two classes of users: Primary Users (PUs) and Secondary Users (SUs). A service unit with a priority queue and another service unit with an orbit are assigned to the PUs and SUs, respectively.

In this work, we focus on the non-reliability of the servers and the collisions at the secondary servers. The primary and secondary servers are subject to random breakdowns and repairs. A collision is introduced at the retrial part of the cognitive radio network. This conflict invokes the interruption of a servicing packet when a new arriving call requests the server unit.

The novelty of the investigation is the non-reliability of servers and the inclusion of conflicts at the secondary server.

By the use of simulation, we analyze the effect of the non-reliability of the servers on the mean response time of the secondary users.
\end{abstract}

Keywords: Finite source queuing systems · Simulation · Cognitive Radio Networks $\cdot$ Performance and reliability measures $\cdot$ Collision $\cdot$ Nonreliable servers

\section{Introduction}

Cognitive radio (CR) has emerged as a promising technology to realize dynamic spectrum access and increase the efficiency of a largely under utilized spectrum. As it was defined in $[1,2]$, the cognitive radio network (CRN) is a network made up of CRs by extending the radio link features to network layer function and above. By means of CRs cooperation, the network is able to sense its environment, learn from the history, and accordingly decide the best spectrum settings.

In other words, cognitive radio allows efficient use of the available spectrum by defining two types of users in wireless networks: licensed and unlicensed users. An unlicensed user (also called secondary user (SU)) can use the spectrum if it is not being used at that time by licensed users (also called primary user $(\mathrm{PU})$ ). When the licensed user appears to use the spectrum, the unlicensed user must find another spectrum to use. see for example [3-5].

(C) Springer International Publishing AG 2017

A. Dudin et al. (Eds.): ITMM 2017, CCIS 800, pp. 194-203, 2017.

DOI: $10.1007 / 978-3-319-68069-9 \_16$ 
In this paper we introduce a finite-source queueing model with two (non independent) frequency channels. The cognitive radio architecture consists of two main networks: The Primary Channel Service (PCS) and Secondary Channel Service (SCS). The PCS refers to the existing network, wherein the primary users (PUs) have got a licensed frequency which does not suffer from overloading. The SCS does not have a license to operate in a licensed frequency. Hence, SCS is designed to work with PCS to provide the capability to utilize or share the unused spectrum in an opportunistic way. The secondary users have got also a frequency band but it suffers from overloading.

In our environment the band of the PUs is modelled by a queue where the requests has preemptive priority over the SUs requests. The band of the SUs is described by a retrial queue: if the band is free when the request arrives then it is transmitted. Otherwise, the request goes to the orbit if both bands are busy. The primary server unit and the secondary server unit are not reliable and are assumed to be subject to breakdown and repair. Also, the retrial part of the cognitive radio network suffered from collision at the secondary server unit, which means that the arriving packets involve into collision with the servicing packets $[6,7]$.

Hence, it should be noted that the novelty of this model is the introduction of the non-reliability of the servers with conflict (collision), and by using simulation, we analyze the effect of the request generation, retrial, service, failure and repair rate of the primary and secondary users on the mean response time of the secondary users.

\section{System Model}

Figure 1 illustrates a finite source queueing system which is used to model the considered cognitive radio network. The queueing system contains two interconnected, not independent sub-systems. The first part is for the requests of the PUs. The number of sources is denoted by $N_{1}$. These sources generate high priority requests with an exponentially distributed inter-request times with the parameter $\lambda_{1}$. The generated requests are sent to a single server unit (Primary Channel Service - PCS). The service times are supposed to be also exponentially distributed with the parameter $\mu_{1}$.

The second part is for the requests of the SUs. There are $N_{2}$ sources, the interrequest times and service times of the single server unit (Secondary Channel Service - SCS) are assumed to be exponentially distributed random variables with rate $\lambda_{2}$ and $\mu_{2}$, respectively.

The servers can be in three states: idle, busy and failed. For the primary server unit, if it is idle, the service of the generated high priority packet starts immediately. If the server is busy with a high priority request, the packet joins the preemptive priority queue. When the unit is engaged with a request from SUs, the service is interrupted and the interrupted low priority task is sent back to the SCS. Depending on the state of secondary channel the interrupted job is directed to either the server or the orbit. The server unit can fail during an idle 


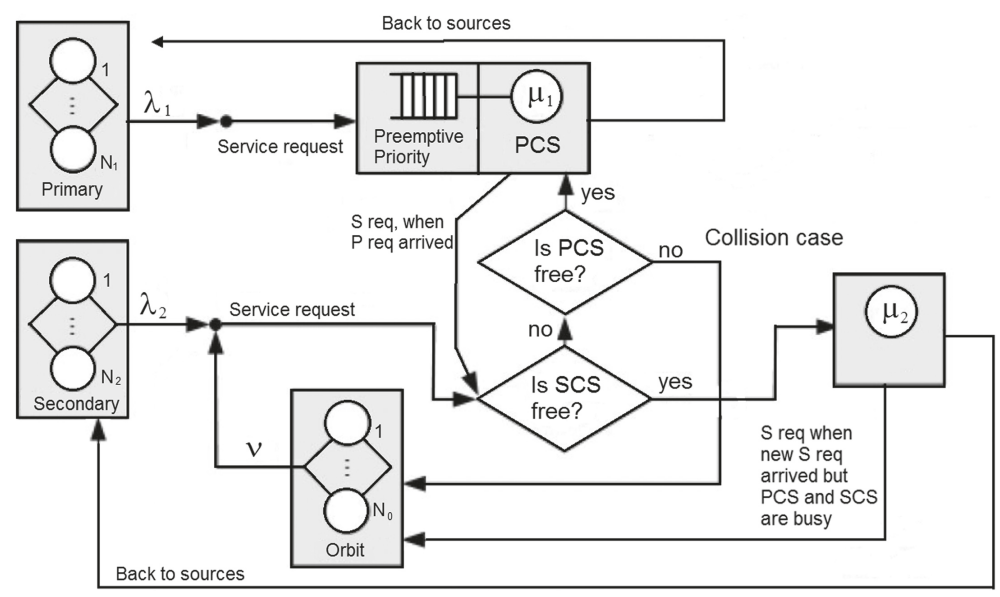

Fig. 1. A priority and a retrial queue with collision

or busy state according to an exponentially distributed time with parameter $\gamma_{1}$. If the server fails in busy state, the service is interrupted and the interrupted request joins the preemptive priority queue. The repair time is exponentially distributed random variable with a parameter $\sigma_{1}$.

In case of requests from SUs. If the SCS is idle, the service starts. If it is busy, the packet looks for the PCS. In case of an idle PCS, the service of the low priority packet begins at the high priority channel (PCS). If the PCS is busy, the packet involves into collision with the low priority servicing packet and both goes to the orbit. the same failure state can occur at the secondary server unit according to an exponentially distributed time with parameter $\gamma_{2}$, the repair time is exponentially distributed with the parameter $\sigma_{2}$. The interrupted packet also goes to the orbit. From the orbit it retries to be served after an exponentially distributed time with parameter $\nu$. All the random variables involved in the model construction are supposed to be independent of each other.

To create a stochastic process describing the behaviour of the system, the following notations are introduced

- $k_{1}(t)$ is the number of high priority sources at time $t$,

- $k_{2}(t)$ is the number of low priority sources at time $t$,

$-q(t)$ denotes the number of high priority requests in the priority queue at time $\mathrm{t}$,

- $o(t)$ is the number of requests in the orbit at time $t$,

$-y(t)=0$ if there is no job in the PCS unit, $y(t)=1$ if the PCS unit is busy with a job coming from the high priority class, $y(t)=2$ when the PCS unit is servicing a job coming from the secondary class at time $\mathrm{t}$

$-c(t)=0$ when the SCS unit is idle and $c(t)=1$, when the SCS is busy at time $\mathrm{t}$. 
It is easy to see that

$$
\begin{gathered}
k_{1}(t)= \begin{cases}N_{1}-q(t), & y(t)=0,2, \\
N_{1}-q(t)-1, & y(t)=1 .\end{cases} \\
k_{2}(t)= \begin{cases}N_{2}-o(t)-c(t), & y(t)=0,1, \\
N_{2}-o(t)-c(t)-1, & y(t)=2 .\end{cases}
\end{gathered}
$$

Since all the random variables involved in the model construction are assumed to be exponentially distributed we could create a continuous time Markov chain with multidimensional state space. However, the main problem is the determination of its stationary distribution. Instead we prefer the stochastic simulation and in a further paper we aim to use non-exponentially distributions and to investigate the effect of distribution of specific random time on different performance measures.

The input parameters are collected in Table 1.

Table 1. List of simulation parameters

\begin{tabular}{l|l|l}
\hline Parameter & Maximum & Value at $t$ \\
\hline Active primary sources & $N_{1}$ & $k_{1}(t)$ \\
\hline Active secondary sources & $N_{2}$ & $k_{2}(t)$ \\
\hline Primary generation rate & & $\lambda_{1}$ \\
\hline Secondary generation rate & & $\lambda_{2}$ \\
\hline Requests in priority queue & $N_{1}-1$ & $q(t)$ \\
\hline Requests in orbit & $N_{2}-1$ & $o(t)$ \\
\hline Primary service rate & & $\mu_{1}$ \\
\hline Secondary service rate & & $\mu_{2}$ \\
\hline Retrial rate & & $\nu$ \\
\hline Primary failure rate & & $\gamma_{1}$ \\
\hline Secondary failure rate & & $\gamma_{2}$ \\
\hline Primary repair rate & & $\sigma_{1}$ \\
\hline Secondary repair rate & & $\sigma_{2}$ \\
\hline
\end{tabular}

\section{Simulation Results}

In order to estimate the mean response times of the requests, the batch mean method is used which is the most popular confidence interval technique for the output analysis of a steady-state simulation, see for example [8-10].

There are many possible combinations of the cases, we considered only the following sample results showing the effect of the non-reliability of the servers on the mean response time of the secondary users. 
Table 2. Numerical values of model parameters

\begin{tabular}{l|l|l|l|l|l|l|l|l|l|l|l}
\hline No & $N_{1}$ & $N_{2}$ & $\lambda_{1}$ & $\lambda_{2}$ & $\mu_{1}$ & $\mu_{2}$ & $\sigma_{1}$ & $\sigma_{2}$ & $\gamma_{1}$ & $\gamma_{2}$ & $\nu$ \\
\hline Figures 2 and 3 & 6 & 6 & 0.6 & $\mathrm{x}-$ axis & 4 & 4 & 1 & 1 & 0.05 & 0.05 & 0.4 \\
\hline Figures 4 and 5 & 6 & 6 & 0.6 & 0.6 & 4 & 4 & 1 & 1 & 0.05 & 0.05 & $\mathrm{x}-$ axis \\
\hline Figures 6 and 7 & 6 & 6 & 0.6 & 0.6 & $\mathrm{x}-$ axis & 4 & 1 & 1 & 0.05 & 0.05 & 0.4 \\
\hline Figure 8 & 10 & 10 & 0.1 & 0.1 & 4 & 4 & $\mathrm{x}-$ axis & $\mathrm{x}-$ axis & 0.05 & 0.05 & 0.4 \\
\hline Figure 9 & 10 & 10 & 0.1 & 0.1 & 4 & 4 & 0.05 & 0.05 & $\mathrm{x}-$ axis & $\mathrm{x}-$ axis & 0.4 \\
\hline Figure 10 & 10 & 10 & 0.1 & 0.1 & 4 & 4 & 0 & $\mathrm{x}-$ axis & 0 & 0.05 & 0.4 \\
\hline
\end{tabular}

For the easier understanding the numerical value of parameters are collected in Table 2.

Figure 2 shows the effect of the request generation rate on the mean response time of the secondary users in the two cases: Secondary server unit non-reliable and both servers non-reliable, where the Fig. 3 shows the same effect in the two cases of non-reliability with collision in the retrial part of the system. Figures show the phenomenon of having a maximum value of the mean response time which was noticed in [11]. The collision involves longer response time for the users as it was expected.

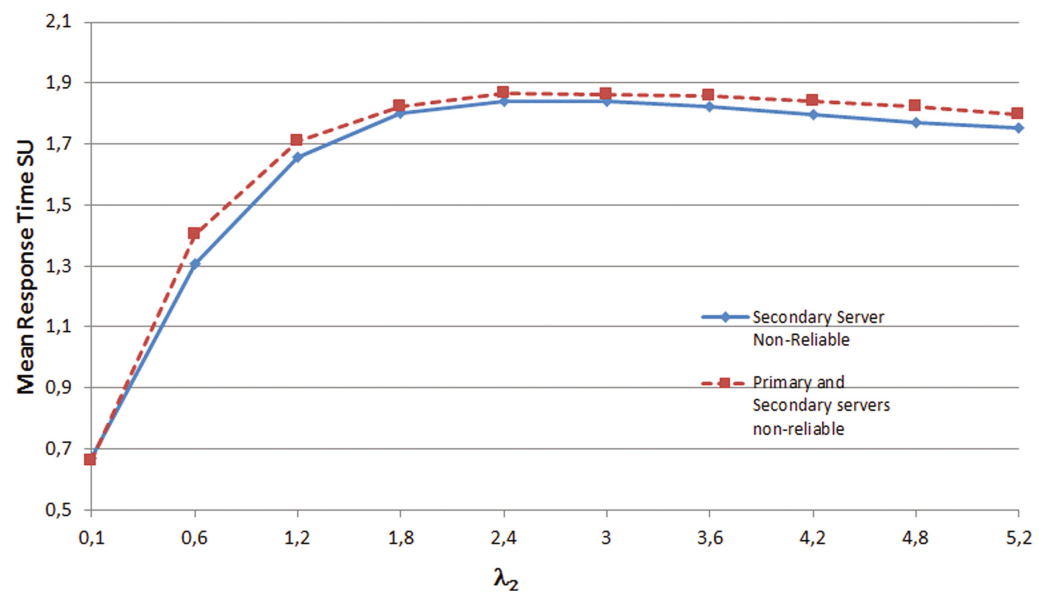

Fig. 2. The effect of servers non-reliability on the mean response time of secondary users vs $\lambda_{2}$

Figures 4 and 5 shows the effect of the retrial rate on the mean response time of the secondary users. In Fig. 4, the non-reliability of the primary server unit does not have any effect on the mean response time of secondary users where the retrial rate is increasing. However, the non-reliability of the primary server has an effect on the mean response time which can be shown on the Fig. 5. It means that in the cognitive radio networks, having a reliable primary server involves 


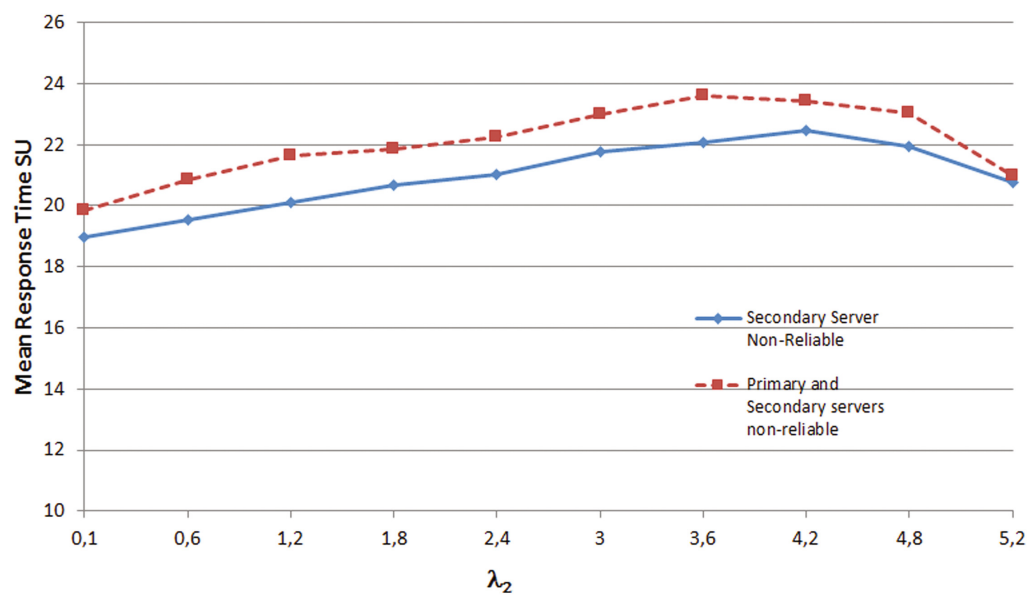

Fig. 3. The effect of servers non-reliability with collision on the mean response time of secondary users vs $\lambda_{2}$

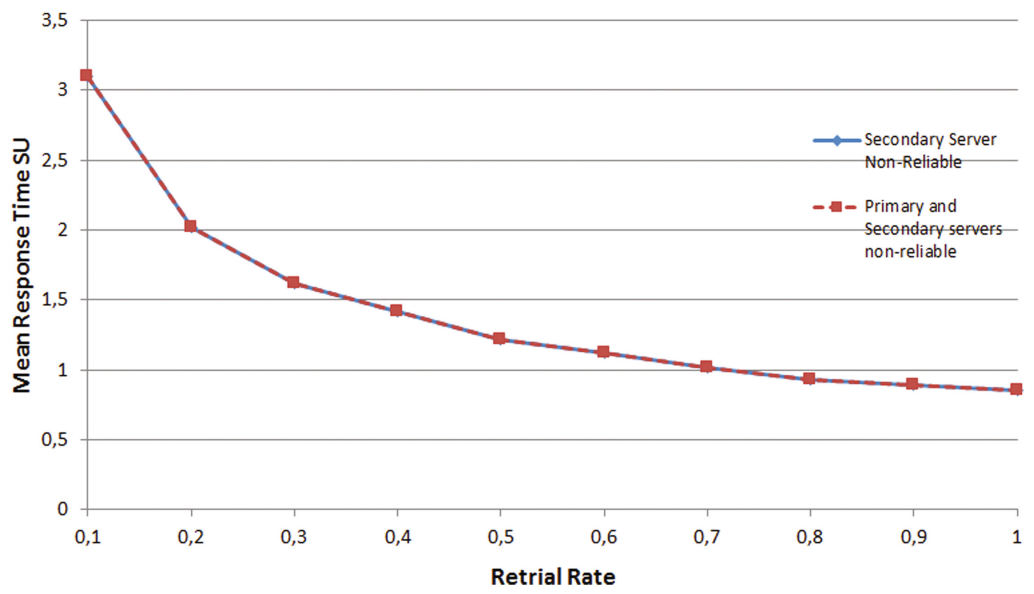

Fig. 4. The effect of servers non-reliability on the mean response time of secondary users vs the retrial rate $\nu$

shortest mean response time of secondary users where there is a collision in the retrial part of the system.

Figures 6 and 7 illustrate the effect of the primary service rate on the mean response time of the secondary users. The non-reliability of the primary server has an effect on the mean response time of the secondary users in the case of the collision where the primary service rate is increasing. A longer response time can be seen in the case of the collision in the retrial part, as it was expected.

Figure 8 shows the effect of the non-reliability of the servers on the mean response time of the secondary users where the repair rate is increasing. 


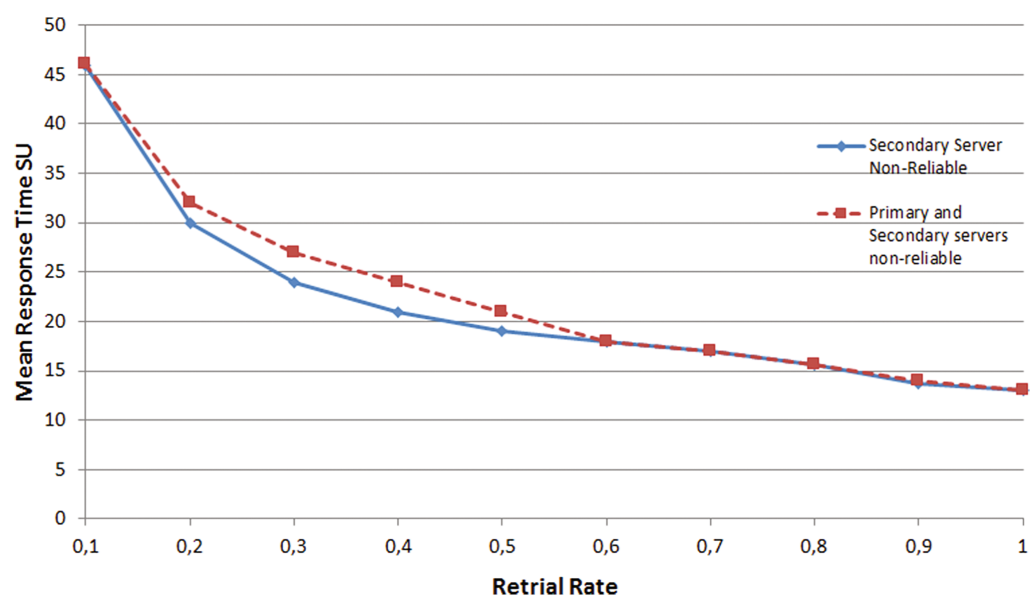

Fig. 5. The effect of servers non-reliability with collision on the mean response time of secondary users vs the retrial rate $\nu$

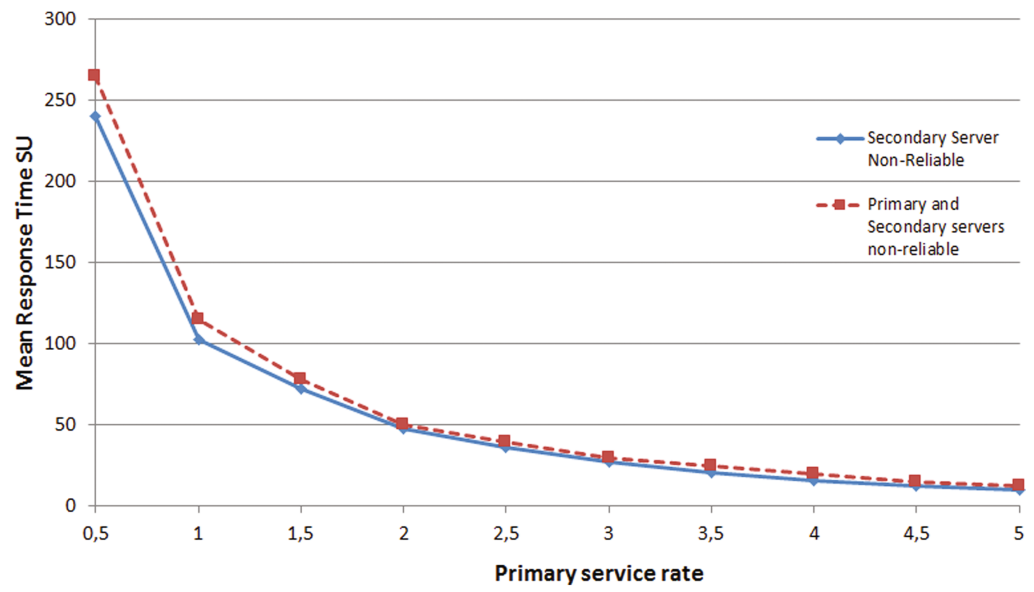

Fig. 6. The effect of servers non-reliability with collision on the mean response time of secondary users vs Primary service rate $\mu_{1}$

The first case is where the primary server is non-reliable, in this case the value of the mean response time of the secondary users becomes a constant when the primary repair rate $\left(\sigma_{1}\right)$ is higher. The second case is where the secondary server is non-reliable, in this case, the value of the mean response time of the secondary users is decreasing when the secondary repair rate $\left(\sigma_{2}\right)$ is increasing.

Figure 9 illustrates the effect of the non-reliability of the servers on the mean response time of the secondary users where the failure rate is increasing. As it was expected, increasing the failure rate involves longer response time in the both cases (primary server non-reliable and secondary server non-reliable). 


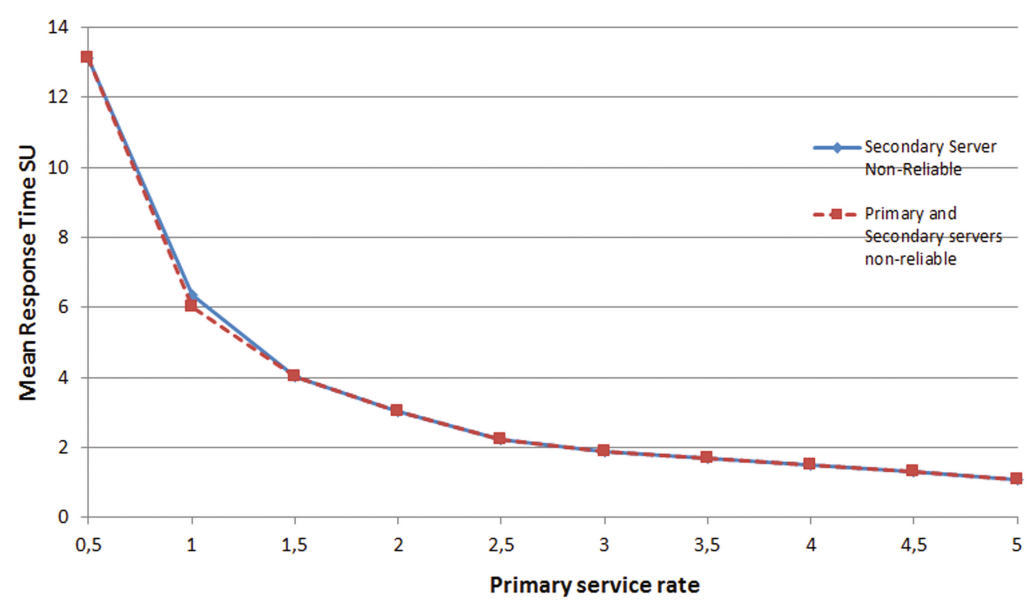

Fig. 7. The effect of servers non-reliability on the mean response time of secondary users vs Primary service rate $\mu_{1}$

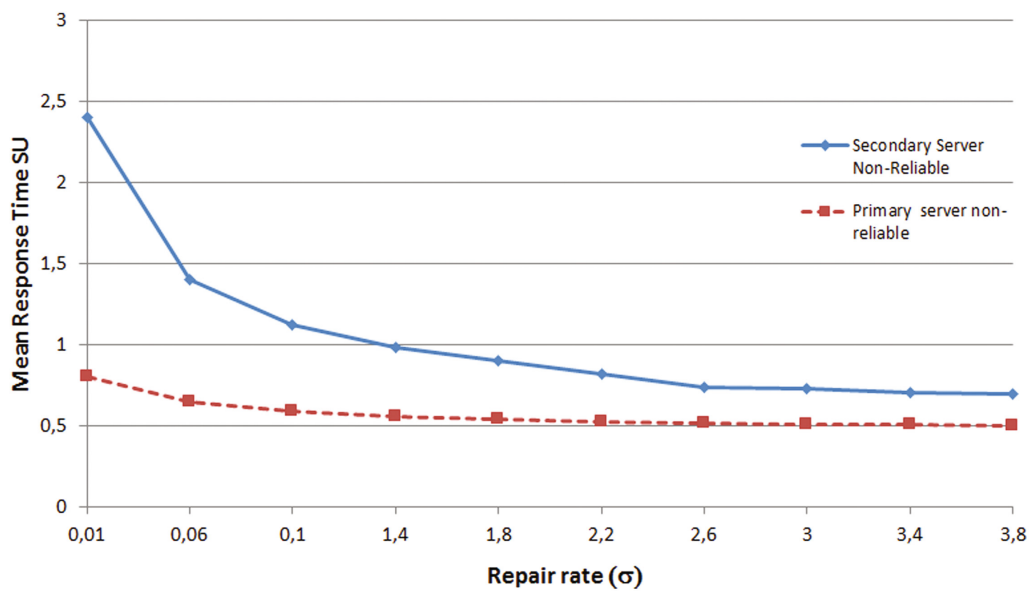

Fig. 8. The effect of servers non-reliability on the mean response time of secondary users vs the repair rate $\sigma$

The last Figure shows the effect of the collision on the mean response time of the secondary users. The conflict on a non-reliable secondary server causes a very long response time. 


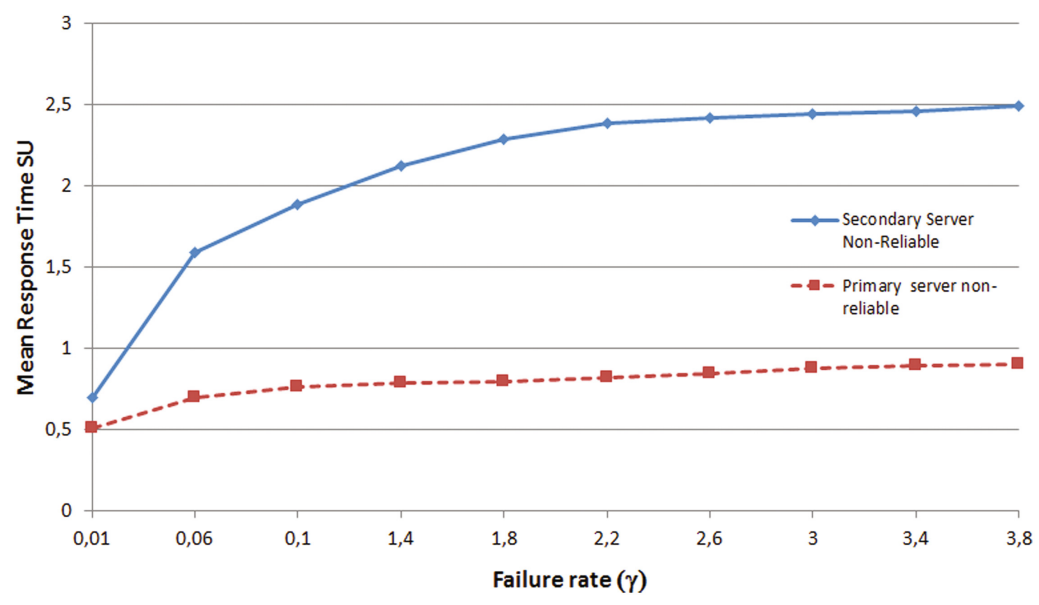

Fig. 9. The effect of servers non-reliability on the mean response time of secondary users vs the failure rate $\gamma$

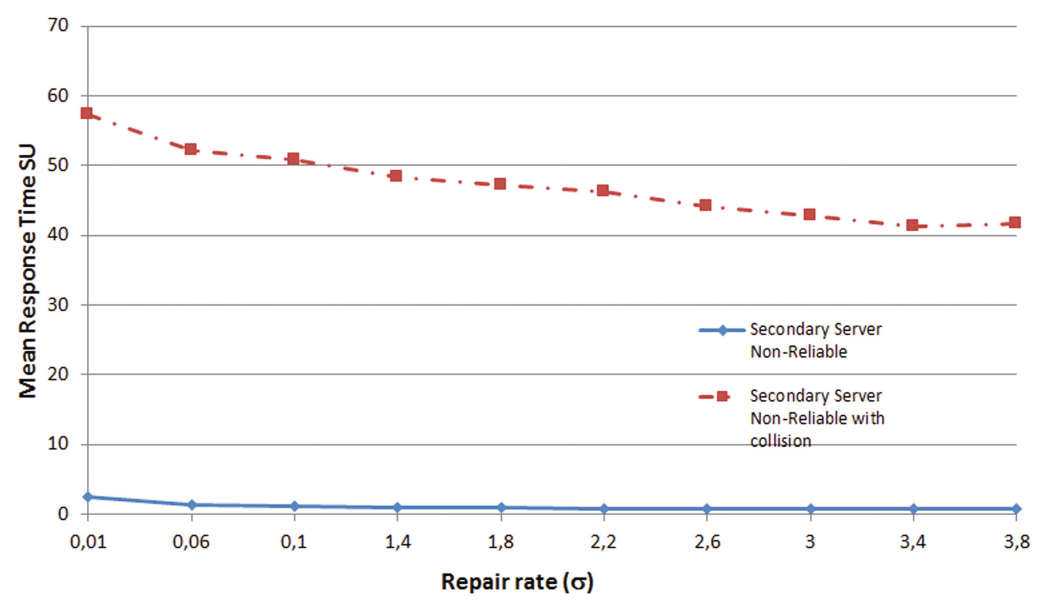

Fig. 10. The effect of servers non-reliability and collision on the mean response time of secondary users vs the repair rate $\sigma$

\section{Conclusions}

In this paper a finite-source retrial queueing model was proposed with two bands servicing primary and secondary users in a cognitive radio network. Primary users have preemptive priority over the secondary ones in servicing at primary channel. At the secondary channel an orbit is installed for the secondary packets finding the server busy upon arrival. The non-reliability and conflict (collision) of the servers were introduced. By using simulation, several sample examples illustrates the effect of the non-reliability of the servers and the collisions at the 
secondary service on the mean response time of the secondary users. This paper is the starting point of a more complex investigation where generally distributed random variables are introduced to see the effect of the distribution of the specific random variable on the main performance measures of the system.

Acknowledgments. The work of Nemouchi H. was supported by the Stipendium Hungaricum Scholarship.

\section{References}

1. Léon, O., Hernández-Serrano, J., Soriano, M.: Securing cognitive radio networks. Int. J. Commun. Syst. 23, 633-652 (2010)

2. Ahmed, H., AlQahtani, S.A.: Performance evaluation of joint admission and eviction controls of secondary users in cognitive radio networks. Arab. J. Sci. Eng. 40, 34469-34481 (2015)

3. Wong, E.W., Foch, C.H., Adachi, F.: Analysis of cognitive radio spectrum decision for cognitive radio networks. IEEE J. Sel. Areas Commun. 29, 757-769 (2011)

4. Zhang, Y., Zhend, J., Chen, H.: Cognitive Radio Networks: Architectures, Protocols, and Standards. CRC Press, Boca Raton (2010)

5. Gao, S., Wang, J.: Performance analysis of a cognitive radio network based on preemptive priority and guard channels. Int. J. Comput. Math. (2014). P.GCOM2013-0621-A

6. Kvach, A., Nazarov, A.: Sojourn time analysis of finite source Markov retrial queueing systems with collision. In: Dudin, A., Nazarov, A., Yakupov, R. (eds.) Information Technologies and Mathematical Modelling - Queueing Theory and Applications. Communications in Computer and Information Science, vol. 564, pp. 64-72. Springer, Cham (2015). doi:10.1007/978-3-319-25861-4_6

7. Nazarov, A., Kvach, A., Yampolsky, V.: Asymptotic analysis of closed Markov retrial queuing system with collision. In: Dudin, A., Nazarov, A., Yakupov, R., Gortsev, A. (eds.) ITMM 2014. CCIS, vol. 487, pp. 334-341. Springer, Cham (2014). doi:10.1007/978-3-319-13671-4_38

8. Law, A.M., Kelton, W.D.: Simulation Modeling and Analysis, 2nd edn. McGrawHill College, New York (1991)

9. Carlstein, E., Goldsman, D.: The use of subseries values for estimating the variance of a general statistic from a stationary sequence. Ann. Stat. 14, 1171-1179 (1986)

10. Sztrik, J., Bérczes, T., Nemouchi, H., Melikov, A.Z.: Performance modeling of finite-source cognitive radio networks using simulation. DCCN 2016. Communications in Computer and Information Science, vol. 678, pp. 64-73. Springer, Cham (2016)

11. Sztrik, J., Roszik, J., Almási, B.: The effect of server's breakdowns on the finitesource retrial queueing systems. In: 6th International Conference on Applied Informatics, vol. 2, pp. 221-230. Eger, Hungary (2004) 